\title{
Polarized Neutron Reflectometry as a Nondestructive Tool for Studies on the Buried Interfaces in Magnetic Thin Films
}

\author{
Masayasu Takeda \\ Quantum Beam Science Directorate (QuBS), Japan Atomic Energy Agency(JAEA), \\ 2-4 Shirane Shirakata, Tokai-mura, Naka-gun, Ibaraki 319-1195, Japan \\ Fax: +81-29-282-5939, e-mail: takeda.masayasu@jaea.go.jp
}

\begin{abstract}
Most magnetic recording devices such as read heads and magnetic or magneto optical recording media have multilayer structures. In these devices, magnetic structural information on the interfaces is the key to achieve the high performance. Polarized neutron reflectometry is a unique and nondestructive tool to determine the magnetic interfacial structures. Recently the neutron reflectometer, SUIREN, was installed at the neutron guide hall in JRR-3 of Japan Atomic Energy Agency (JAEA). This reflectometer is capable of polarized neutron reflectometry, and is now open to users.
\end{abstract}

Key words: polarized neutron, reflectometer, magnetic structure, magnetic device, buried interface

\section{INTRODUCTION}

$\mathrm{X}$-ray and neutron reflectometries have been now widely used to determine the depth profile of thin films and artificial multilayers. These two techniques give similar information on the internal layered structures. As compared with $\mathrm{X}$-ray, neutron has several advantages: 1 . most materials are transparent for neutrons, 2 . the nuclear scattering length (scattering power) for neutrons is different even for adjacent elements in the periodic table, 3. neutrons are sensitive to the difference of isotopes, 4 . neutrons have the interaction between their spin and the magnetic moments in the materials.

The available neutrons in the present neutron facilities are, however, much less than photons in the synchrotron radiation facilities. In addition to this, both probes, X-ray and neutron, are complementary to each other because X-ray sees the electron cloud around nuclei, while neutron sees the nuclei themselves, and observes the electron cloud only via the magnetic interactions. Therefore the complementary use of both X-ray and neutron reflectometries is very useful and sometimes necessary for the investigation of magnetic multilayers.

Magnetic recording technique is now indispensable to our digital life style. The recording equipment such as hard disk drives consists of several magnetic devices that have layered structures. In the devices the minute magnetic structures at the interfaces are the key to achieve the high performance. Thus it is essential to acquire the magnetic structural information in the vicinity of interfaces to develop the high performance devices. It is, however, difficult to study such detailed internal structures using the conventional methods because the interfaces are buried inside the multilayers.

Polarized neutron reflectometry (PNR) is a unique and nondestructive tool to investigate the buried internal magnetic structures. Specular neutron reflectivity gives us the information on the depth profile of the multilayers, including magnetic interfacial roughness. In addition, the lateral internal structural information can be obtained by off-specular reflection. In this article, brief introduction of the polarized neutron specular reflectivity and a new reflectometer installed at JRR-3 of Japan Atomic Energy Agency (JAEA) is given.

\section{POLARIZED NEUTRON REFLECTIVITY[1]}

In calculating the neutron reflectivity, the incident, reflected and transmitted neutrons are treated as plane waves. The nuclear and magnetic potential affect only component of wave vector of incident neutron normal to the reflection plane, $k_{0}(=k \sin \theta)$, where $k$ is the wave vector of incident neutron and $\theta$ is half the scattering angle. Thus the reflectivity calculation is equivalent to one-dimensional square well potential problem as shown in Fig. 1.

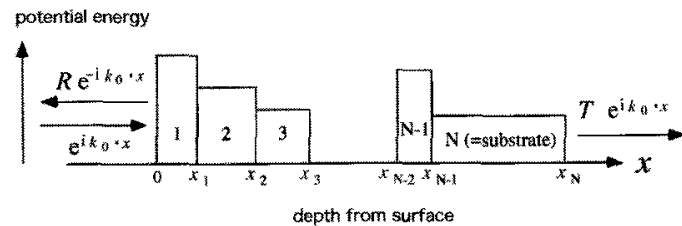

Fig. 1 One dimensional square well potential equivalent to problem of neutron reflection from multilayers.

In this figure, the multilayer consists of $N$ layers (regions). The regions 0 and $N+1$ are vacuum, and region $N$ is a substrate. Each interface is denoted as $x_{i}$ $(i=0,1, \ldots, N)$. The plane wave of incident neutron, reflected and transmitted neutrons are written as $\exp (+i$ $\left.k_{0} x\right), R \exp \left(-i k_{0} x\right)$, and $T \exp \left(+i k_{0} x\right)$, respectively. The reflectivity of the multilayer is defined by $|R|^{2}$.

When neutrons interact magnetic moments, $\boldsymbol{M}$, in the magnetic multilayers, neutron is affected only by the component of $\boldsymbol{M}$ normal to the scattering vector, $\boldsymbol{M}_{\mathrm{n}}$. In other words, neutron is insensitive to component of $\boldsymbol{M}$ parallel to the scattering vector. In 
addition, there is another selection rule; neutron spin is flipped by the component of $M_{\mathrm{n}}$ normal to the spin, $M_{\perp}$, but the spin state is not change by the component of $\boldsymbol{M}_{\mathrm{n}}$ parallel to the spin, $M_{\| /}$.

The Shrödinger equation for the plane wave in the potential, $U_{j}$, in the region $j$ is

$$
-\frac{\hbar^{2}}{2 m} \nabla^{2} \Phi+U_{j} \Phi=E \Phi
$$

In the case of magnetic multilayers, $U_{j}$ consists of nuclear and magnetic potentials, and is written as

$U=\frac{\hbar^{2}}{2 \pi m} \rho(b \pm p)$

where $\rho$ is the density, $b$ the nuclear scattering length, and $p$ the magnetization (magnetic scattering length). The suffix of $j$ is omitted in this equation.

In the case of the polarized neutrons, the two spin states must be distinguished, and the two spin states are mixed due to $M_{\perp}$. Thus Eq. (1) is modified as

$$
\begin{aligned}
& \nabla^{2} \Phi_{+}+\left[k_{0}^{2}-\left(b^{\prime}+B_{/ /}\right)\right] \Phi_{+}-B_{\perp} \Phi_{-}=0 \\
& \nabla^{2} \Phi_{-}+\left[k_{0}^{2}-\left(b^{\prime}-B_{/ /}\right)\right] \Phi_{-}-B_{\perp} \Phi_{+}=0
\end{aligned}
$$

Here $\Phi_{ \pm}$is the wave function of each spin state, + or -, and $b^{\prime}, B_{/ /}$and $B_{\perp}$ are the nuclear scattering length, the parallel and normal components of magnetic flux density induced by $\boldsymbol{M}_{\mathrm{n}}$, in this order. These values are scaled such that Eq. (3) is satisfied with the scaled values. The general solutions of Eq. (3) are

$$
\begin{gathered}
\Phi_{+}=C_{1} \exp \left(i k_{+} x\right)+C_{2} \exp \left(-i k_{+} x\right) \\
+C_{3} \exp \left(i k_{-} x\right)+C_{4} \exp \left(-i k_{-} x\right)
\end{gathered}
$$

and

$$
\begin{gathered}
\Phi_{-}=D_{1} \exp \left(i k_{+} x\right)+D_{2} \exp \left(-i k_{+} x\right) \\
+D_{3} \exp \left(i k_{-} x\right)+D_{4} \exp \left(-i k_{-} x\right),
\end{gathered}
$$

where $k_{ \pm}$are the normal component of wave vectors of neutrons in the medium with the two spin states, + or -. Using Eqs. (3) and (4), the following relations are deduced.

$$
-k_{+}^{2}+k_{0}^{2}-b^{\prime}= \pm \sqrt{B_{/ /}^{2}+B_{\perp}^{2}}= \pm B_{T},
$$

and

$$
k_{ \pm}^{2}=k_{0}^{2}-b^{\prime} \pm B_{T}=n^{2} k_{0}^{2}
$$

Here $n$ is the reflective index of the $j$-th layer. $\Phi_{-}$can be written using Eq. (5) as

$$
\begin{gathered}
\Phi_{-}=\frac{B_{/ /}-B_{T}}{B_{\perp}} C_{1} \exp \left(i k_{+} x\right)+\frac{B_{/ /}-B_{T}}{B_{\perp}} C_{2} \exp \left(-i k_{+} x\right) \\
+\frac{B_{/ /}+B_{T}}{B_{\perp}} C_{3} \exp \left(i k_{-} x\right)+\frac{B_{/ /}+B_{T}}{B_{\perp}} C_{4} \exp \left(-i k_{-} x\right)
\end{gathered}
$$

Calculation of reflectivity is now equivalent to determination of the coefficients, $C_{1}, C_{2}, C_{3}$ and $C_{4}$ at each interface.

At the start, the interface between vacuum and semi-infinite layer is considered. When the wave function of neutron with each spin state in the vacuum is written as $\varphi_{ \pm}$, the boundary conditions at the surface are

$$
\left\{\begin{array}{l}
\Phi_{ \pm}(x=0)=\varphi_{ \pm}(x=0) \\
\Phi_{ \pm}^{\prime}(x=0)=\varphi_{ \pm}^{\prime}(x=0)
\end{array}\right. \text {. }
$$

Using the boundary conditions, $\Phi_{ \pm}(x)$ can be expressed as matrix formula;

$$
\left(\begin{array}{c}
\Phi_{+}(x) \\
\Phi_{+}^{\prime}(x) \\
\Phi_{-}(x) \\
\Phi_{-}^{\prime}(x)
\end{array}\right)=\left(\begin{array}{llll}
a_{11} & a_{12} & a_{13} & a_{11} \\
a_{21} & a_{11} & a_{23} & a_{13} \\
a_{13} & a_{14} & a_{33} & a_{34} \\
a_{23} & a_{13} & a_{43} & a_{33}
\end{array}\right)\left(\begin{array}{c}
\varphi_{+}(0) \\
\varphi_{+}^{\prime}(0) \\
\varphi_{-}(0) \\
\varphi_{-}^{\prime}(0)
\end{array}\right) .
$$

Each matrix element is

$$
\begin{aligned}
& a_{11}=a_{22}=\frac{B_{T}+B_{\| /}}{2 B_{T}} \cos \left(k_{+} x\right)+\frac{B_{T}-B_{/ /}}{2 B_{T}} \cos \left(k_{-} x\right) \\
& a_{12}=\frac{B_{T}+B_{/ l}}{2 B_{T}} \frac{1}{k_{+}} \sin \left(k_{+} x\right)+\frac{B_{T}-B_{\| /}}{2 B_{T}} \frac{1}{k_{-}} \sin \left(k_{-} x\right) \\
& a_{13}=a_{24}=a_{31}=a_{42}=-\frac{B_{\perp}}{2 B_{T}}\left\{\cos \left(k_{+} x\right)-\cos \left(k_{-} x\right)\right\} \\
& a_{14}=a_{32}=-\frac{B_{\perp}}{2 B_{T}}\left\{\frac{1}{k_{+}} \sin \left(k_{+} x\right)-\frac{1}{k_{-}} \sin \left(k_{-} x\right)\right\} \\
& a_{21}=-\frac{B_{T}+B_{/ /}}{2 B_{T}} k_{+} \sin \left(k_{+} x\right)-\frac{B_{T}-B_{/ /}}{2 B_{T}} k_{-} \sin \left(k_{-} x\right) \\
& a_{23}=a_{41}=-\frac{B_{\perp}}{2 B_{T}}\left\{k_{+} \sin \left(k_{+} x\right)-k_{-} \sin \left(k_{-} x\right)\right\} \\
& a_{33}=a_{44}=\frac{B_{T}-B_{\| /}}{2 B_{T}} \cos \left(k_{+} x\right)+\frac{B_{T}+B_{/ /}}{2 B_{T}} \cos \left(k_{-} x\right) \\
& a_{34}=\frac{B_{T}-B_{/ /}}{2 B_{T}} \frac{1}{k_{+}} \sin \left(k_{+} x\right)+\frac{B_{T}+B_{/ /}}{2 B_{T}} \frac{1}{k_{-}} \sin \left(k_{-} x\right) \\
& a_{43}=-\frac{B_{T}-B_{\| /}}{2 B_{T}} k_{+} \sin \left(k_{+} x\right)-\frac{B_{T}+B_{/ /}}{2 B_{T}} k_{-} \sin \left(k_{-} x\right)
\end{aligned}
$$

If the sample has the internal layered structure instead of the semi-infinite layer, the wave functions in adjacent layers must be satisfied with the boundary condition equivalent to Eq. (8) at each interface, $x=x_{j}(j=0,1, \ldots, N)$. When the 4 by 4 matrix, $A 0(x)$, in Eq. (9) corresponding to the $j$-th interface is 
denoted by $A^{j} \equiv A^{j}\left(x_{j}-x_{j-1}\right)$, the wave functions at the $j$-th interface hold the following relation.

$$
\left(\begin{array}{c}
\Phi_{+}\left(x_{j}\right) \\
\Phi_{+}^{\prime}\left(x_{j}\right) \\
\Phi_{-}\left(x_{j}\right) \\
\Phi_{-}^{\prime}\left(x_{j}\right)
\end{array}\right)=A^{j} \cdots A^{2} A^{1}\left(\begin{array}{c}
\varphi_{+}(0) \\
\varphi_{+}^{\prime}(0) \\
\varphi_{-}(0) \\
\varphi_{-}^{\prime}(0)
\end{array}\right)
$$

If the incident neutrons are perfectly polarized to the + spin state, the wave functions can be written as

$$
\begin{aligned}
& \Phi_{+}\left(x_{N} \leq x\right)=T_{++} \exp \left(i k_{0} x\right) \\
& \varphi_{+}(x \leq 0)=\exp \left(i k_{0} x\right)+R_{++} \exp \left(-i k_{0} x\right) \\
& \Phi_{-}\left(x_{N} \leq x\right)=T_{+-} \exp \left(i k_{0} x\right) \\
& \varphi_{-}(x \leq 0)=R_{+-} \exp \left(-i k_{0} x\right)
\end{aligned}
$$

Here $T_{++}$and $R_{++}$are transmittance and reflectance with the non-spin-flip (nsf) process, and $T_{+-}$and $R_{++}$are those with the spin-flip (sf) one. The reflectivities with nsf and sf processes can be calculated as $\left|R_{+++}\right|^{2}$ and $\left|R_{+-}\right|^{2}$, respectively. Using Eq. (11) and (12), the following equation is deduced.

$$
\begin{aligned}
& \left(\begin{array}{c}
T_{++} \exp \left(i k_{0} x_{N}\right) \\
i k_{0} T_{++} \exp \left(i k_{0} x_{N}\right) \\
T_{+} \exp \left(i k_{0} x_{N}\right) \\
i k_{0} T_{+-} \exp \left(i k_{0} x_{N}\right)
\end{array}\right) \\
& =\left(\begin{array}{llll}
A_{11} & A_{12} & A_{13} & A_{14} \\
A_{21} & A_{22} & A_{23} & A_{24} \\
A_{31} & A_{32} & A_{33} & A_{34} \\
A_{41} & A_{42} & A_{43} & A_{44}
\end{array}\right)\left(\begin{array}{c}
1+R_{++} \\
i k_{0}\left(1-R_{++}\right) \\
R_{+} \\
-k_{0} R_{+}
\end{array}\right)
\end{aligned}
$$

where $A_{i m}$ are the matrix elements of $A^{N} \cdots A^{2} A^{1}$ in Eq. (11). The final forms of $R_{++}$and $R_{+}$are

$$
R_{++}=\frac{1}{|A|}\left|\begin{array}{cccc}
A_{11}+i k_{0} A_{12} & e_{N} & -A_{13}+i k_{0} A_{14} & 0 \\
A_{21}+i k_{0} A_{22} & i k_{0} e_{N} & -A_{23}+i k_{0} A_{24} & 0 \\
A_{31}+i k_{0} A_{32} & 0 & -A_{33}+i k_{0} A_{34} & e_{N} \\
A_{41}+i k_{0} A_{42} & 0 & -A_{43}+i k_{0} A_{44} & i k_{0} e_{N}
\end{array}\right|
$$

and

$$
R_{+-}=\frac{1}{|A| \mid}\left|\begin{array}{cccc}
-A_{11}+i k_{0} A_{12} & e_{N} & A_{11}+i k_{0} A_{12} & 0 \\
-A_{21}+i k_{0} A_{22} & i k_{0} e_{N} & A_{21}+i k_{0} A_{22} & 0 \\
-A_{31}+i k_{0} A_{32} & 0 & A_{31}+i k_{0} A_{32} & e_{N} \\
-A_{41}+i k_{0} A_{42} & 0 & A_{41}+i k_{0} A_{42} & i k_{0} e_{N}
\end{array}\right| .
$$

In the above formula, $e_{N}$ is substituted for $\exp \left(-i k_{0} x_{N}\right)$, and $|A|$ is determinant of matrix, $A^{N} \cdots A^{2} A^{1}$. The similar formula for incident neutrons perfectly polarized to the - spin state are calculated in the same manner.

$$
R_{-+}=\frac{1}{|A|}\left|\begin{array}{cccc}
A_{13}+i k_{0} A_{14} & e_{N} & -A_{13}+i k_{0} A_{14} & 0 \\
A_{23}+i k_{0} A_{24} & i k_{0} e_{N} & -A_{23}+i k_{0} A_{24} & 0 \\
A_{33}+i k_{0} A_{34} & 0 & -A_{33}+i k_{0} A_{34} & e_{N} \\
A_{43}+i k_{0} A_{44} & 0 & -A_{43}+i k_{0} A_{44} & i k_{0} e_{N}
\end{array}\right|,
$$

and

$$
R_{--}=\frac{1}{|A|}\left|\begin{array}{cccc}
-A_{11}+i k_{0} A_{12} & e_{N} & A_{13}+i k_{0} A_{14} & 0 \\
-A_{21}+i k_{0} A_{22} & i k_{0} e_{N} & A_{23}+i k_{0} A_{24} & 0 \\
-A_{31}+i k_{0} A_{32} & 0 & A_{33}+i k_{0} A_{34} & e_{N} \\
-A_{41}+i k_{0} A_{42} & 0 & A_{43}+i k_{0} A_{44} & i k_{0} e_{N}
\end{array}\right| .
$$

These four different reflectivities, $\left|R_{++}\right|^{2},\left|R_{++}\right|^{2}$, $\left|R_{-+}\right|^{2}$ and $\left|R_{-}\right|^{2}$ can be measured by polarized neutron reflectometers with neutron spin analyzer, while two kinds of reflectivity, $\left|R_{+}\right|^{2}=\left|R_{++}\right|^{2}+\left|R_{+}\right|^{2}$ and $\left|R_{-}\right|^{2}=\left|R_{-}\right|^{2}+\left|R_{-+}\right|^{2}$ are obtained by those without the analyzer.

\section{NEW NEUTRON REFLECTOMETER, SUIREN, \\ AT JRR-3 OF JAEA}

We installed the polarized neutron reflectometer, PORE, at the pulsed neutron facility, KENS, of High Energy Accelerator Research Organization (KEK) in Tsukuba and have been studying the magnetic multilayers since 1991. The second reflectometer, ARISA, was also installed at KENS as a dedicated reflectometer for investigation polymer science particularly on free surface and interface in 2001.

However, KENS, has been permanently shut down at the end of March 2006 because the first neutron beam of Materials and Life Science Facility of Japan Proton Accelerator Complex (J-PARC) is expected in 2008. Consequently, we had only one neutron reflectometer, MINE, at JRR-3 after the shutdown of KENS in Japan, and it has been operated not as a dedicated reflectometer but as a multipurpose neutron spectrometer [2].

Under the situation, a new reflectometer, SUIREN (Apparatus for Surface and Interface Investigations

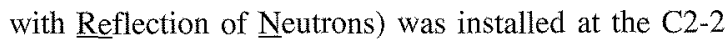
cold neutron beam port in the neutron guide hall of JRR-3 [3]. SUIREN was specially designed to enable to switch the vertical and horizontal sample geometry; neutrons are reflected in the horizontal plane by a sample placed vertically at the sample position, or reflected in the vertical plane by a sample settled horizontally. Although the later horizontal geometry is necessary for the investigation of free surface and interface, the mechanism of the instruments for the horizontal geometry is more complicated than that for the vertical one. Thus SUIREN was completed as the vertical reflectometer at the first stage. Layout of SUIREN is schematically drawn in Fig. 3, and the basic instrumental parameters are summarized in Table 1. 


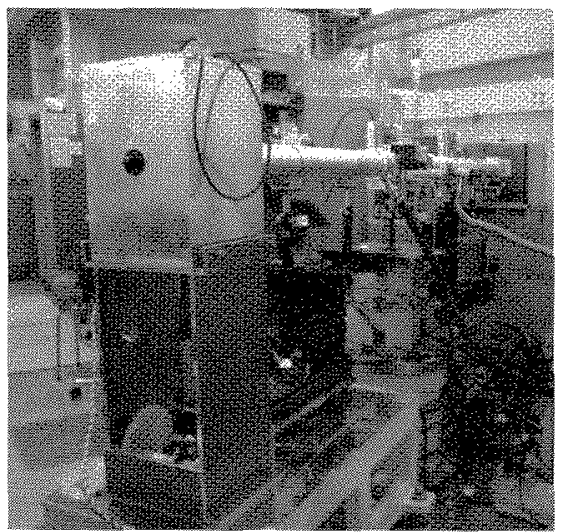

Fig. 2 New neutron reflectometer, SUIREN, installed at the $\mathrm{C} 2-2$ beam hole of the $\mathrm{C} 2$ cold neutron guide of JRR-3 in JAEA.

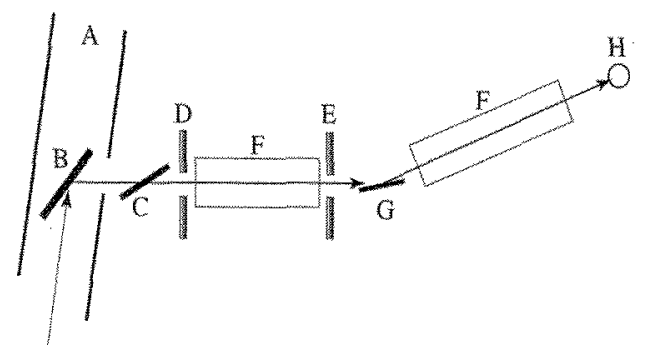

Fig. 3 Instrument layout of SUIREN. A: the C2 cold neutron guide, $\mathrm{B}$ : $\mathrm{PG}(002)$ monochromator, $\mathrm{C}: \mathrm{PG}$ filter, D: slit 1 (S1), E: slit 2 (S2), F: evacuated flight tube or neutron polarizer ( analyzer ), G: sample, and $\mathrm{H}$ : detector.

Table 1. Instrument characteristics

$\begin{array}{lc}\text { wavelength } & 0.382 \mathrm{~nm} \\ \text { wavelength resolution } & 1.3 \% \\ \text { maximum beam size } & 20 \mathrm{~mm} \text { (width) } \\ & 80 \mathrm{~mm} \text { (height) } \\ \text { distance between two slits, } & 1450 \mathrm{~mm} \\ \text { S1 and } \mathrm{S} 2 & \\ \text { sample-detector distance } & 1100 \mathrm{~mm} \\ \text { the number of incident neutrons } & 2.5 \times 10^{4} \mathrm{n} / \mathrm{s} / \mathrm{cm}^{2} \\ \text { ( } \Delta \theta= \pm 0.04 \text { degree) } & \\ \text { typical background } & 4.5 \times 10^{-3} \mathrm{cps}\end{array}$

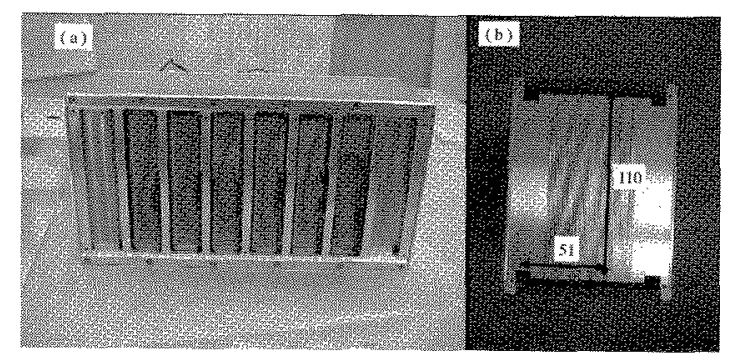

Fig. 4 Polarizing device made of remanent polarizing supermirrors for SUIREN. (a) side view and (b) cross section.
In the polarized neutron reflectivity measurements, the flight tube ( $\mathrm{F}$ in Fig. 3) is replaced by a polarizing device. At the moment only a polarizer is available. The polarizing device was assembled by Swiss Neutronics. The polarizer consists of neutron polarizing remanent supermirrors and electromagnets that choose the spin state of polarized neutron (Fig. 4). Thus the conventional spin flipper is unnecessary, and the device is called "spin selector" [4].

Figure 5 shows the first data of polarized neutron reflectivities measured by SUIREN very recently. The sample was an exchange bias multilayer whose structure can be written as $\mathrm{Ta} / \mathrm{Ru} / \mathrm{CoFe} / \mathrm{MnIr} / \mathrm{Ru} / \mathrm{Ta} / \mathrm{SiO}_{2}$ on a $\mathrm{Si}$ substrate. The saturation field of $2 \mathrm{kOe}$ was applied in the sample plane during the measurements. In this figure, $\left|R_{+}\right|^{2}$ is plotted by solid squares and $\left|R_{-}\right|^{2}$ by solid circles. Since the difference between $\left|R_{+}\right|^{2}$ and $\left|R_{-}\right|^{2}$ is clearly observed, this figure shows that SUIREN works correctly as the polarized neutron reflectometer. The neutron spin analyzer will be equipped to perform the polarization analysis (the complete sets of $\left|R_{++}\right|^{2},\left|R_{+-}\right|^{2},\left|R_{-+}\right|^{2}$ and $\left.\left|R_{--}\right|^{2}\right)$ in the near future.

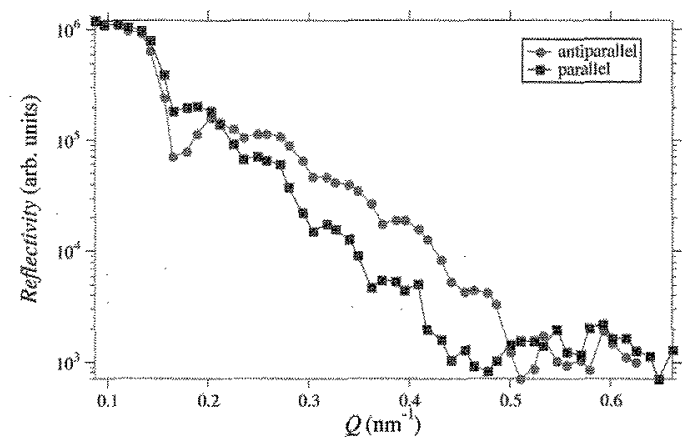

Fig. 5 Neutron spin dependent reflectivities from an exchange bias multilayer.

\section{Acknowledgments}

We are much indebted to M. Tsunoda of Tohoku University for sample preparation of the exchange bias multilayer. Polarized neutron options were introduced to SUIREN in collaboration with $D$. Yamazaki, K. Soyama, A. Birumachi, K. Endoh, and K. Kakurai of JAEA. This work was supported by Japan Society for the Promotion of Science, Grantsin-Aid for Scientific Research.

\section{References}

[1] G.P. Felcher, R. O. Hilleke, R. K. Crawford,

J. Haumann, R. Kleb and G. Ostrowski, Rev. Sci. Instrum, 58, 609-619 (1986).

[2] MINE was installed and is maintained by Kyoto University Research Reactor Institute (KUR).

[3] D. Yamazaki et al., JAEA-Technology 2007 in press (in Japanese).

[4] J. Stahn, M. Christensen, P. Kailbauer, and D. Clemens, PSI-Scientific Report 2001 / Volume III, Chap. 8.

(Received December 9, 2006; Accepted February 12, 2007) 